\title{
A Digital Pre-Distortion Based on Nonlinear Autoregressive With Exogenous Inputs
}

\author{
Pooria Varahram, John Dooley, Keith Finnerty, and Ronan Farrell
}

\begin{abstract}
In this letter, a new pre-distortion technique for power amplifiers in wideband applications is proposed. The proposed pre-distortion technique is based on Nonlinear Autoregressive with Exogenous inputs (NARX). The forward path of the proposed predictive method is based on the memory polynomial. Experimental validation is carried out with 4 carrier WCDMA signal with $20 \mathrm{MHz}$ bandwidth and PAPR $=9.8 \mathrm{~dB}$. The results show significant reduction in the number of coefficients with comparable performance in terms of adjacent channel leakage ratio (ACLR) and error vector magnitude (EVM) to Volterra series techniques.
\end{abstract}

Index Terms-Memory effects, memory polynomial, NARX, power amplifier (PA), Volterra series.

\section{INTRODUCTION}

$\mathbf{P}$ OWER amplifiers (PAs) are one of the pivotal components of wireless communication systems. Although ideal amplification should be linear, the actual PA normally deviates from linear operation. As a result of the nonlinear behavior, the spectral regrowth produced leads to superfluous radiations and adjacent channel interference. Digital pre-distortion (DPD) is one of the most common solutions used to compensate for the non-linearity in the PA. It allows cost-efficient non-linear PAs to run in their non-linear regions with minimized distortions. Until now the majority of the research into this solution relied on the Volterra series [1], [2] and subsets of the Volterra series such as Hammerstein [3], Hammerstein-Wiener [4], and memory polynomial [5] to model the digital pre-distortion.

Despite the Volterra series offering a usable model of the PA with memory effects, it is fundamentally limited by its structure, as a large number of coefficients are required to accurately model a nonlinear PA. A Nonlinear Autoregressive with eXogenous inputs (NARX) which is a special case of Nonlinear Autoregressive moving Average with eXogenous inputs (NARMAX) with no noise dependent model was presented in [6]. In nonlinear systems, Volterra series expands the current output as a series of current and past inputs only. This in turn leads to an explosion in the number of terms to be estimated. In contrast, NARX can easily capture these effects due to having nonlinear lagged terms. By this way the identification of the nonlinear system becomes easier as fewer terms are needed to model the system. Despite the fact that NARX provides

Manuscript received November 9, 2015; revised January 19, 2016; accepted February 2, 2016. Date of publication April 22, 2016; date of current version May 6, 2016. This work was supported by the Science Foundation Ireland under Grant SFI10/CE/I1853.

The authors are with the Callan Institute, Department of Electronic Engineering, National University of Ireland, Kildare, Ireland (e-mail: pooria.varahram@ nuim.ie).

Digital Object Identifier 10.1109/LMWC.2016.2549178 better accuracy in modeling, added care must be taken to ensure stability, compared to Volterra series techniques.

An analogous comparison would be Finite Impulse Response (FIR) filter which comprises the systems present and past inputs. An Infinite Impulse Response (IIR) filter depends on the present and past inputs and also the past outputs. Even though both can be designed to have a similar transfer characteristic, the infinite impulse response filter can be implemented using fewer weights and thus multiplications.

Previous publications have started to explore the use of NARX structures to model or perform DPD for nonlinear PAs. Authors in [7] claimed that Nonlinear Autoregressive with eXogenous inputs can be used to linearize GaN class F PAs using an indirect learning architecture. The proposed three-layer implementation consists of input layer, hidden layer, and output layer. Two identical NARX networks represent the static and dynamic blocks to linearize memory effects and memoryless effects, respectively. In [7], the authors use the NARX structure to linearize the PA; a Levenberg-Marquardt training algorithm is used to calculate the coefficients for the neural network to implement the digital pre-distortion.

Authors in [8] proposed a NAMRA DPD solution for RF PAs. The structure of the DPD in [8] generates an output signal from a lookup table based on the complex gain function. While it achieves the required DPD performance for the given standard, a comparison with other DPD methods such as memory polynomial and generalized memory polynomial (GMP) [9] are not provided.

In this letter we present a novel NARX based DPD solution for RF PAs. Validation of the improved performance of the NARX DPD presented in this letter is demonstrated by experimental measurement. ACLR, EVM, and NMSE values are calculated to give a comprehensive comparison between the proposed approach and the popular GMP and MP techniques.

\section{NARX PRE-Distortion TEChNIQUE}

\section{A. Identification Algorithm}

Here, the identification is based on post-distortion algorithm. Once the post-distortion coefficients have been identified, it can be copied to the running path where the actual pre-distortion is implemented. It has been shown in [10] that the coefficients obtained from post-distortion are identical to pre-distortion and hence can be applied to the running path. The identification procedure is to obtain the coefficients of the inverse of the PA and copy these coefficients to the forward path. The good thing about the NARX DPD is its identification can be done directly using Least Square (LS) estimation. This is in contrast to NARMAX where its coefficients need to be estimated in an iterative way [6]. The method to extract these coefficients is to 
first capture the input and output signals, swap them, and then the least square algorithm needs to be applied as

$$
\begin{aligned}
& u[n]=-\sum_{k=1}^{K} \sum_{i=1}^{I} a_{k i} u[n-i]|u[n-i]|^{k-1} \\
& \quad+\sum_{k=1}^{K} \sum_{m=0}^{M} b_{k m} y[n-d-m]|y[n-d-m]|^{k-1}+e[n]
\end{aligned}
$$

where $u[n]$ and $y[n]$ are the sampled observations of the input and output of the PA, respectively, and $a$ is the delay sample between the input and output of the PA and sometimes called the dead time. It should be noted that, in almost all the literatures, this delay is not considered in their analytical derivation. That is because the propagation delay on an experimental test bench can be accounted for prior to signal analysis; $e[n]$ is the noise sequence which represents the modelling error and all the noise in the system; $a, b$ are the identification parameters and $K$ is the nonlinearity order (odd and even), and $I$ and $M$ are the memory length of the output and input samples, respectively. For the $N$ input-output data sequence, the post-distortion function (1) in matrix form are

$$
u=\Phi w+e
$$

where $\Phi=\left[A_{1}, \ldots, A_{K I}, B_{1}, \ldots, B_{K M}\right]$ is a basis function with $A_{l}=-\sum_{k=1}^{K} \sum_{i=1}^{I} u[n-i]|u[n-i]|^{k-1}$ and $B_{l}=\sum_{k=1}^{K}$ $\sum_{m=0}^{M} y(n-m)|y(n-m)|^{k-1}, w$ is a vector of coefficient $w=\left[a_{11}, \ldots, a_{K I}, b_{10}, \ldots, b_{K M}\right]^{T}, u=[u[1], u[2], \ldots u[N]]^{T}$ where $N$ is the length of the signal and the symbol $(\cdot)^{T}$ denotes complex conjugate transpose. The least square solution to identify the coefficient $w$ is given by

$$
\hat{w}=\left(\Phi^{H} \Phi\right)^{-1} \Phi^{H} u
$$

where $(\cdot)^{H}$ and $(\cdot)^{-1}$ denote Hermitian transpose and inverse of the matrix, respectively, and $\hat{w}$ is the estimated coefficients.

There is no difficulty to extract the estimated coefficients of pre-distortion function $(\hat{w})$, as both sequences of input and output data samples are available from the PA measurement or during the identification procedure. For simplicity, the error term in (1) has been ignored. The main consideration, however, is to apply these coefficients to the pre-distortion function in the running path. The reason is that, in the NARX, the past output samples of the pre-distortion function are not known. Hence, the difficulty of applying NARX DPD is in the running path where the initial output value is not known.

\section{B. New Prediction Method}

Here, a new method to apply the NARX pre-distortion in the running path is proposed. In this approach, the output of the pre-distortion function can be initialized by a few known output values. This depends on the order of the NARX DPD. In this method, the system performance works well even with the first order. The known value can be obtained either from the measurement or, in our case, from the memory polynomial series as follows:

$$
u[n]=\sum_{k=1}^{K} \sum_{m=0}^{M} b_{k m} x[n-m]|x[n-m]|^{k-1}
$$

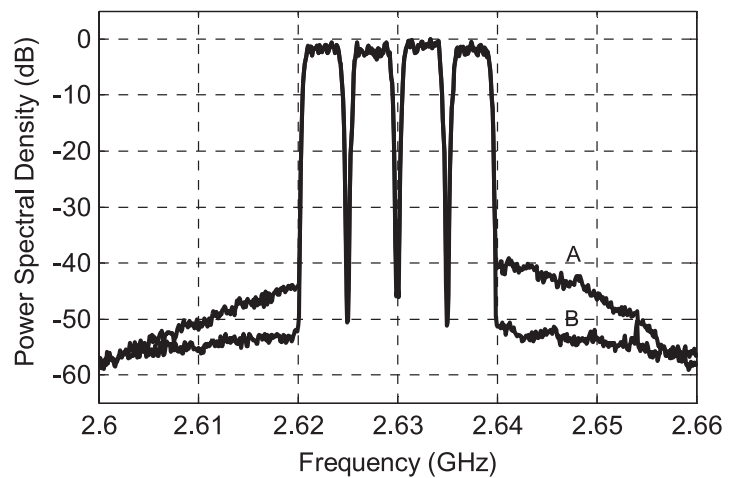

Fig. 1. ACLR performance of the NARX DPD. (A) Output without DPD. (B) Output with NARX.

where $x$ and $u$ are the input and output of pre-distortion, respectively. Hence, the proposed prediction algorithm for the special case of $I=1, M=0$ is as follows:

$$
\left\{\begin{aligned}
u[1]= & \sum_{k=1}^{K} b_{k 0} x[1]|x[1]|^{k-1} \\
u[2]= & -\sum_{k=1}^{K} a_{k 1} u[1]|u[1]|^{k-1}+\sum_{k=1}^{K} b_{k 0} x[2]|x[2]|^{k-1} \\
u[3]= & -\sum_{k=1}^{K} a_{k 1} u[2]|u[2]|^{k-1}+\sum_{k=1}^{K} b_{k 0} x[3]|x[3]|^{k-1} \\
\vdots & \vdots \\
u[N]= & -\sum_{k=1}^{K} a_{k 1} u[N-1]|u[N-1]|^{k-1} \\
& +\sum_{k=1}^{K} b_{k 0} x[N]|x[N]|^{k-1} .
\end{aligned}\right.
$$

The only drawback with this approach is, following the initialization, only the predicted values of the output are used in the right hand-side, hence, any errors in the predicted value quickly accumulate and diverge the series and result in instability. In [11], the generalized frequency response function of the NARX is derived whereby the bounded input bounded output (BIBO) stability is guaranteed by the $\mathrm{BI}$ and $\mathrm{BO}$ spectrum. For the proposed method, the prediction mean square error (MSE) of $\hat{u}(n)$

$$
\operatorname{MSE}\{\hat{u}(n)\}=E\left\{|\hat{u}(n)-u(n)|^{2}\right\}
$$

where $E\{\cdot\}$ is the expected value and $\hat{u}(n)$ is the estimated pre-distortion output. The stability condition can be obtained when the NARX series is converged based on the inequality condition $\|y\|_{2} \leq C\|u\|_{2}$ where $\|\cdot\|_{2}$ operator is the second order norm, which also represents the energy of a signal, and $C$ is a finite value. For the post-distortion algorithm above, the stability condition can be obtained by

$$
\sum_{l=1}^{N}|e(l)|^{2} \leq \delta
$$

where $\delta$ is a small tolerance value. 
TABLE I

NMSE COMPARISON OF DIFFERENT DPD TECHNIQUES

\begin{tabular}{|c|c|c|}
\hline Technique & No. of Coefficients & NMSE (dB) \\
\hline \multicolumn{3}{|l|}{$\overline{\mathrm{MP}}$} \\
\hline$(K=5, M=3)$ & 20 & -45.09 \\
\hline$(K=5, M=5)$ & 30 & -45.41 \\
\hline$(K=10, M=3)$ & 40 & -46.35 \\
\hline$(K=10, M=9)$ & 100 & -47.06 \\
\hline \multicolumn{3}{|l|}{$G M P$} \\
\hline$\left(\begin{array}{l}K, K_{a}, K_{b}=5, M=3 \\
M_{a}, L_{a}, M_{b}, L_{b}=1\end{array}\right)$ & 30 & -45.61 \\
\hline$\left(\begin{array}{l}K, K_{a}, K_{b}=5, M=5 \\
M_{a}, L_{a}, M_{b}, L_{b}=1\end{array}\right)$ & 40 & -46.23 \\
\hline$\left(\begin{array}{l}K, K_{a}, K_{b}=10, M=3 \\
M_{a}, L_{a}, M_{b}, L_{b}=1\end{array}\right)$ & 60 & -46.71 \\
\hline$\left(\begin{array}{l}K, K_{a}, K_{a}=10, M=9 \\
M_{a}, L_{a}, K_{b}, M_{b}, L_{b}=1\end{array}\right)$ & 120 & -47.47 \\
\hline $\begin{array}{l}\text { NARX } \\
(K=5, M=3 I=1)\end{array}$ & 25 & -46.63 \\
\hline$(K=10, M=3, I=1)$ & 50 & -47.59 \\
\hline
\end{tabular}

\section{EXPERimental VAlidation}

The experimental verification and comparison of the NARX DPD is carried out in terms of normalized mean square error (NMSE), ACLR, and EVM. Hence, first NMSE is analyzed followed by ACLR and EVM measurement. The signal used in this experiment is a 4 carrier WCDMA with $20 \mathrm{MHz}$ bandwidth centered at $2.63 \mathrm{GHz}$ with instantaneous-to-average power ratio $(\mathrm{IAR})=9.8 \mathrm{~dB}$ at $0.01 \% \mathrm{CCDF}$. The sampling rate is 92.16 Mbps and 24,576 samples have been captured for training DPD. The test bench includes an $R \& S$ vector signal generator SMU200A and R\&S FSQ vector signal analyzer, Doherty PA (RTP26010-N1) with typical gain of $40 \mathrm{~dB}$. LabVIEW and Matlab are used for signal generation, measurement, and processing, and the resulting signal is supplied directly to the PA from the SMU200A. The captured signal was averaged eight times to suppress the noise floor of the signal.

Endeavoring to demonstrate the effectiveness of the proposed DPD solution in as fair a comparison as possible, the optimum model dimensions are sought for both the memory polynomial (MP) and generalized memory polynomial (GMP) models. Fig. 1 shows the ACLR performance of the proposed NARX DPD. The ACLR performance of other techniques is shown in Table II. It can be observed that there is asymmetry in the out of band distortion which is due to the memory effects and is compensated by applying NARX DPD. The difference is more obvious on the left side of the spectrum. Table I shows the NMSE comparison between MP, GMP, and NARX. From Table I, it can be seen that NARX DPD gives comparable NMSE performance against MP and GMP with fewer number of coefficients. This reduces the computational complexity compared to MP and GMP techniques. However, with the same complexity, the NARX DPD outperforms MP and GMP in terms of NMSE performance. It is important to mention that a higher value of auto regressive $(I)$ does not have much im-
TABLE II

PERFormanCE COMPARISON OF DifFERENT DPD TECHNIQUES

\begin{tabular}{lccc}
\hline \hline Technique & $\begin{array}{c}\text { ACLR (dBc) } \\
(\mathrm{UB} / \mathrm{LB})\end{array}$ & EVM (\%) [2] & POP (W) \\
\hline No DPD & $-40.9 /-44.1$ & 14.41 & 28.8 \\
MP & $-50.7 /-51.8$ & 2.51 & 28.8 \\
GMP & $-51.2 /-52.5$ & 1.48 & 28.8 \\
NARX & $-51.3 /-53.9$ & 1.2 & 28.8 \\
\hline
\end{tabular}

provement in the NMSE performance. Table II gives the performance comparison between different DPD techniques in terms of ACLR and EVM metrics with the peak output power (POP) of 28.8 Watt. It can be seen that NARX with 25 coefficients outperforms MP and GMP with 30 coefficients.

\section{CONCLUSION}

In an effort to reduce the computational and memory overhead for pre-distortion approaches, a novel NARX-based DPD is presented. The proposed approach shows marginally better linearization performance compared to memory polynomial and generalized memory polynomial techniques. However, the true benefit of this approach is in its accuracy for reduced numbers of coefficients. Experimentally validated performance comparisons have been made in this letter by providing the analysis of the ALCR and EVM.

\section{REFERENCES}

[1] A. Zhu, J. Dooley, and T. J. Brazil, "Simplified volterra series based behavioral modeling of RF power amplifiers using deviation-reduction," in Proc. IEEE MTT-S Int. Dig., Jun. 2006, pp. 1113-1116.

[2] P. B. Kenington, High-Linearity RF Amplifier Design. Boston, MA, USA: Artech House, 2000

[3] H. W. Kang, Y. S. Cho, and D. H. Youn, "On compensating nolinear distortions of an OFDM system using efficient adaptive predistorter," IEEE Trans. Commun., vol. 47, pp. 522-526, Apr. 1999.

[4] E. W. Bai, "An optimal two stage identification algorithm for Hammerstein-Wiener nonlinear systems," in Proc. Amer. Control Conf., Jun. 1998, pp. 2756-2760.

[5] J. Kim and K. Konstantinou, "Digital predistortion of wideband signals based on power amplifier model with memory," Electron. Lett., vol. 37, pp. 1417-1418, Nov. 2001.

[6] S. A. Billings and I. J. Leontaritis, "Identification of nonlinear systems using parametric estimation techniques," in Proc. IEEE Conf. Control Appl., Warwick, U.K., 1981, pp. 183-187.

[7] L. Aguilar-Lobo, A. Garcia-Osorio, J. Loo-Yau, S. Ortega-Cisneros, P. Moreno, J. Rayas-Sanchez, and J. Reynoso-Hernandez, "A digital predistortion technique based on a narx network to linearize GaN class $\mathrm{F}$ power amplifiers," in Proc. IEEE 57th Int. Midwest Symp. Circuits Syst. (MWSCAS), Aug. 2014, pp. 717-720.

[8] P. L. Gilabert, G. Montoro, and A. Cesari, "A recursive digital predistorter for linearizing RF power amplifiers with memory effects," in Proc. APMC'06, Pacifico Yokohama, Japan, Dec. 2006, vol. 2, pp. 1043-1047.

[9] R. D. Morgan, Z. Ma, J. Kim, M. G. Zierdt, and J. Pastalan, "A generalized memory polynomial model for digital predistortion of RF power amplifiers," IEEE Trans. Signal Process., vol. 54, no. 10, pp. 3852-3860, Oct. 2006.

[10] M. Schetzen, The Volterra and Wiener Theories of Nonlinear Systems. New York, NY, USA: Wiley, 1980.

[11] X. Jing and Z. Lang, Frequency Domain Analysis and Design of Nonlinear Systems Based on Volterra Series Expansion, Understanding Complex Systems. New York, NY, USA: Springer-Verlag, 2015. 